\title{
Clinical and Radiological Analysis of Children and Adolescents With Tuberculosis in Bahia, Brazil
}

\author{
Rosana Franco, Maria Angélica Santana, \\ Eliana Matos, Virgínia Sousa and \\ Antônio Carlos M. Lemos
}

\begin{abstract}
Children's Outpatient Pneumology Department, Octávio Mangabeira Hospital, Salvador, BA, Brazil
\end{abstract}

\begin{abstract}
We reviewed the clinical and radiological characteristics of tuberculosis (TB) in children and adolescents at the Hospital Especializado Octávio Mangabeira, (HEOM) in Salvador, Bahia. This study included 275 TB patients aged 1 to 15 years seen between January 1990 and November 2001. Standardized forms were filled out on the basis of a review of patient records and $x$-rays. Through a retrospective and descriptive analysis, it was found that $51.6 \%$ were male, $35.3 \%$ were aged 1 to 5 years, $28 \%$ were aged 6 to 10 and $36.7 \%$ were aged 11 to 15 . Among all patients, 79.6\% lived in the city of Salvador. A history of contact with TB was found in 63.9\%, most frequently among children under 5 years old; $77.2 \%$ were vaccinated with Bacillus CalmetteGuerin (BCG). The most frequently observed symptoms were coughing (76\%), fever $\mathbf{7 3 . 1 \% )}$ weight loss $(53.1 \%)$, and $4.7 \%$ were asymptomatic. Pulmonary TB was most frequent $(57.8 \%)$ and extra-pulmonary TB occurred in $24.4 \%$, with a predominance of hilar adenopathy. Both forms occurred simultaneously in $17.8 \%$. In $53.1 \%$ of the cases the diagnosis was not determined by bacteriology or pathological anatomy; in these cases diagnosis was reached through clinical and radiological criteria, contact history, a tuberculin test $\geq 10 \mathrm{~mm}$ and a positive response to tuberculostatic drugs.

Key Words: Tuberculosis, children, radiology, chest x-ray.
\end{abstract}

Despite the advances of science, we have entered the third millennium with eight million new cases of TB annually worldwide [1]. In Brazil, conditions still persist that lead to the spread of this disease: chaotic urban growth, malnutrition, precarious housing, unemployment, inadequate health service and the HIV epidemic. The high endemicity of this disease favors early contact with the bacillus during a stage of life when

Received on 20 May 2002; revised 27 December 2003.

Address for correspondence: Dr. Rosana Franco. Rua do Escravo Miguel, 198, Apto 502 - Ondina, 40170-000. Salvador, Bahia, Brazil. Phone: 5571 237-0457 / Fax: 5571 261-0278.

E-mail: rosanafranco@uol.com.br

This study was conducted at the Children's Outpatient Pneumology Department, "Hospital Especializado Octávio Mangabeira". Support: NUPEP- "Núcleo de Pesquisa em Pneumologia". Tuberculosis Reference Center - State of Bahia Department of Health

The Brazilian Journal of Infectious Diseases 2003;7(1):73-81 (C) 2003 by The Brazilian Journal of Infectious Diseases and Contexto Publishing. All rights reserved. the immune system is immature [2]. As a result, children are susceptible to contagion and are at a high risk of developing serious forms of the disease. The best form of prevention is vaccination with BCG [3].

TB in children has different characteristics from those of post-primary tuberculosis in adults. Diagnosis is the most difficult aspect of examining TB in children because in its primary form, it is paucibaccillary, and bacteriological corroboration is nearly always lacking [2-4]. In clinical practice, child TB cases are diagnosed on the basis of clinical and radiological criteria, contact history and the tuberculin test [4-5].

\section{Materials and Methods}

A retrospective study was conducted on a series of 275 children and adolescents aged 1 to 15 years, residing in Bahia and seen at the outpatient Children's Pneumology Department of the HEOM, a TB Reference Center in the State of Bahia, between 
January, 1990, and November, 2001. The study included a review of patient records and chest $\mathrm{x}$-rays, and patient data was recorded on standardized forms.

This outpatient clinic treats children and adolescents with a wide range of respiratory diseases from the entire state of Bahia. Within this population, we investigated patients with clinical and radiological status suggestive of TB, and those that fulfilled the criteria for TB diagnosis were included in the study.

The clinical and radiological criteria for suspecting TB in children are: 1) a history of contact with TB sufferers within the last two years; 2) clinical status compatible with TB (cough, fever and weight loss), slow progress or failure to respond to antibiotics; 3 ) reactive tuberculin test: $\geq 10 \mathrm{~mm}$ in patients not vaccinated with BCG or vaccinated over two years before, and $\geq 15$ $\mathrm{mm}$ in those vaccinated less than two years before; [34]4) chest $x$-rays that suggest primary or post-primary tuberculosis. Radiological criteria were indispensable for the diagnosis of pulmonary forms of TB [2-5].

Criteria for confirming TB diagnosis included one or more of the following: 1) direct analysis of acid-fast bacilli (AFB) positive sputum; 2) culture positive for M. tuberculosis from a biological specimen (sputum, bronchoalveolar wash, pleural fluid, liquor and others); 3) histopathological examination of biopsy material (ganglion, pleura, skin, bronchi and lungs) showing a chronic granulomatous inflammatory reaction with caseous necrosis and negative fungal stain; 4) favorable therapeutic response to Isoniazid + Rifampicin + Pyrazinamide progressing to cure. Although available at the hospital, bronchoscopy was not routinely used in the diagnosis of patients with AFB-negative sputum smears.

None of the patients included in the study had been revaccinated with BCG.

The following variables were analyzed: 1) Demographics and origins: age, gender, city/town and neighborhood and/or sanitary districts, 2) Clinical: cough, fever, weight loss, chest pain, dyspnea, bloody sputum, peripheral adenomegaly; epidemiological history of recent contact (less than or equal to two years) with tuberculosis sufferers; presence of BCG vaccination scar on the right arm (at the lower insertion of the deltoid muscle) and classification of the clinical forms of the disease: primary, post-primary and extrapulmonary; 3) Laboratory: Tuberculin testing with PPD Rt 232 UT, bacilloscopy and $M$. tuberculosis culture conducted with Loewenstein-Jensen medium using sputum, bronchial wash, bronchoalveolar wash, gastric wash, aspirated ganglion fluid, pleural fluid, liquor or tissue fragments; 4) Radiological: Chest x-rays (posterior-anterior and lateral) to detect enlarged mediastinal lymph nodes, pulmonary infiltrates, atelectasis, cavitations and pleural effusion, as well as bone x-rays showing osteolytic lesions; 5) Anatomic Pathology: Histopathological examination of biopsy material showing chronic granulomatous inflammatory reaction with caseous necrosis in tissue fragments.

The statistical analysis was basically descriptive. Categorical variables were described through proportions. The software used was SPSS 9.0 for Windows.

\section{Results}

We found that $51.6 \%$ of the patients were male and were distributed in the following age groups: $35.5 \%$ aged 1 to 5 years, $28 \%$ aged 6 to 10 years and $36.7 \%$ aged 11 to 15 years. Among all patients, $79.6 \%$ lived in the city of Salvador and $61.2 \%$ (134/219) were from the low-income population and lived in the peripheral areas of the city.

Among the patients, 8.4\% (23/275) did not provide information on history of recent contact with $\mathrm{TB}$ carriers. Among the rest, 63.9\% (161/252) reported recent contact. Among these individuals, contact was more frequent among those 1 to 5 years old, $44.0 \%$ (71/161), than among patients 6 to 10 and 11 to 15 ; each of these two latter groups were found at frequencies of $28.0 \%$ (45/161). In $36.1 \%$ of the cases (91/252), there was no history of contact.

In $29.8 \%$ of the cases, the records gave no information about $B C G$ vaccination (82/275). Among the rest, the vaccination scar was found in $77.2 \%$ of patients (149/ $193)$ and $22.8 \%$ (44/193) did not have the scar (not vaccinated) (Table 1); 79.5\% of the unvaccinated patients (35/44) came from the city of Salvador. 
Table 1. Characteristics of patients

\begin{tabular}{lrr}
\hline Characteristics & N & \% \\
\hline Age - years $(\mathrm{n}=275)$ & 97 & \\
$1-5$ & 77 & 35.3 \\
$6-10$ & 101 & 28.0 \\
$11-15$ & 142 & 36.7 \\
Gender $(\mathrm{M}=275)$ & & 51.6 \\
Place of Origin & 219 & \\
$\quad$ Capital & 56 & 79.6 \\
Countryside & & 20.4 \\
Recent contact with tuberculosis $(\mathrm{n}=252)$ & 161 & 63.9 \\
$\quad$ Yes & 91 & 36.1 \\
$\quad$ No & & \\
Vaccination with BCG $(\mathrm{n}=193)$ & 149 & 77.2 \\
$\quad$ Yes & 44 & 22.8 \\
No &
\end{tabular}

Table 2. Distribution of tuberculosis patients according to their pulmonary involvement $(n=275)$

\begin{tabular}{lrc}
\hline Classification & N & \% \\
\hline Exclusively pulmonary & 159 & 57.8 \\
Exclusively extra-pulmonary & 67 & 24.4 \\
Pulmonary + extra-pulmonary & 49 & 17.8 \\
\hline
\end{tabular}

The most frequent symptoms were coughing in $76 \%$ of the cases (209/275), fever in $73.1 \%$ (201/275) and weight loss in $53.1 \%$ (146/275). Hemoptysis was the least frequent symptom, occurring in $12.4 \%$ of the cases $(34 / 275)$, and $4.7 \%$ of the patients were asymptomatic (13/295), diagnosed by investigating their contact history.

The exclusively pulmonary forms of the disease were the most frequent, found in $57.8 \%$ of the cases; exclusively extra-pulmonary forms occurred in $24.4 \%$ and both forms were simultaneously associated in $17.8 \%$ (Table 2).

The most frequently observed pulmonary form was primary evolving TB (mediastinal lymph node, mediastinal lymph node-pulmonary, pneumonic and bronchial) which occurred in 50.9\% of the cases (140/ 275). The most frequently observed extra-pulmonary form was peripheral lymph node tuberculosis, found in $21.5 \%$ of the cases $(59 / 275)$, and $21.8 \%$ of the patients (60/275) had multiple episodes, with more than one disease site involved, including pulmonary + extrapulmonary, or more than one form of extra-pulmonary tuberculosis at the same time (Table 3).

The proportion of patients that were examined with the tuberculin test was $83.6 \%$ (230/275); $82.7 \%$ of these were positive reactors $>10 \mathrm{~mm}(190 / 230)$. Among reactors 57\% (131/230) produced reactions $>15 \mathrm{~mm}, 13.5 \%$ were non-reactors with 0 to $4 \mathrm{~mm}$ (31/230), and $3.9 \%$ had weak reactions of 5 to $9 \mathrm{~mm}$ (9/230). 
Table 3. Distribution of tuberculosis lesions among all involved sites recorded $(\mathrm{n}=275)$

\begin{tabular}{lrr}
\hline Clinical forms/sites involved & N & \% \\
\hline Pulmonary & 208 & 75.6 \\
Primary progressive & 140 & 50.9 \\
Mediastinal lymph nodes & 56 & 20.4 \\
Mediastinal-pulmonary lymph nodes & 49 & 17.8 \\
Pneumonic & 34 & 12.4 \\
Bronchial & 1 & 0.4 \\
Post-primary & 68 & 24.7 \\
Extra-pulmonary & 136 & 49.5 \\
Peripheral lymph nodes & 59 & 21.5 \\
Pleural effusion & 36 & 13.1 \\
Skin & 13 & 4.7 \\
Miliary & 12 & 4.4 \\
Osteoarticular & 11 & 4.0 \\
Meningeal & 4 & 1.5 \\
Renal & 1 & 0.4 \\
Multipleinvolvement** & 60 & 21.8 \\
\hline
\end{tabular}

* 17 patients had two or more extra-pulmonary forms simultaneously.

** Patients with involvement of more than one site of the disease simultaneously and it could be pulmonary + extra-pulmonary or more than one extra-pulmonary form.

Table 4. Distribution of the findings in abnormal chest x-rays according to the sites of lesions ( $\mathrm{n}=237$ )

\begin{tabular}{lrr}
\hline Sites of lesions & N & \% \\
\hline Bilateral & 73 & 30.8 \\
Right unilateral & 110 & 46.4 \\
Left unilateral & 54 & 22.8 \\
\hline
\end{tabular}

Table 5. Distribution of diagnostic methods used in cases with confirmation of tuberculosis $(n=129)$

\begin{tabular}{lrr}
\hline Diagnostic method & N & \% \\
\hline Biopsy compatible exclusively & 59 & 45.7 \\
Positive bacilloscopy exclusively & 49 & 38.0 \\
Positive culture for $M$. tuberculosis exclusively & 9 & 7.0 \\
More than one method simultaneously & 12 & 9.3 \\
\hline
\end{tabular}


Chest $\mathrm{x}$-rays were available for $97.8 \%$ of the patients (269/275) and 2.2\% who had the extrapulmonary form of the disease $(6 / 275)$ were not $x$ rayed. Among all the x-rays, $11.9 \%$ were normal (32/ 269); all patients had extra-pulmonary tuberculosis, except for one who had bronchial TB.

Among the chest x-rays with abnormal results, $30.8 \%$ had bilateral lesions, $46.4 \%$ had right unilateral lesions and $22.8 \%$ had left unilateral lesions (Table 4). Among the abnormal radiograms, the most common findings were (in order of frequency): alveolar infiltrate in 56.5\% (134/237), hilar/mediastinal adenopathy in $44.3 \%$ (105/237), interstitial infiltrate in 27\% (64/275), pleural effusion in $15.6 \%$ (37/237), cavitary lesions in $14.8 \%(35 / 237)$, atelectasis in 5.5\% (13/275) and miliary infiltrate in $5.1 \%(12 / 275)$. These abnormalities occurred separately or in association in the same patient.

Among the methods used for diagnosis, bacilloscopy was not used in $35.6 \%$ of the patients. Bacilloscopy was positive in $32.8 \%$ (58/177) and negative in $67.2 \%$ (119/177). Among those with positive bacilloscopy of the sputum, the frequency was higher in individuals aged 11 to 15 years, that is, $84.5 \%$ $(49 / 58)$ than in those aged 1 to 10 years, with a frequency of $15.5 \%(9 / 58)$.

An $M$. tuberculosis culture was made for only $22.1 \%(61 / 275)$ of the patients, and it was positive in $31.1 \%$ of these cases $(19 / 61)$. Histopathological examinations were made for $23.6 \%$ of the patients $(65 /$ 275). Among these, $93.8 \%$ (61/65) had the extrapulmonary form of the disease and $6.2 \%(4 / 65)$ had the pulmonary form. All biopsied cases were compatible with tuberculosis.

Among the patients studied, 53.1\% (146/275) were diagnosed following the criteria for TB, through a favorable response to tuberculostatic drugs (Group I), and $46.9 \%$ (129/275) had their diagnosis confirmed through bacteriological and histopathological methods (Group II).

In Group I, 70.5\% of cases (103/146) had the pulmonary forms of the disease, $10.9 \%(16 / 146)$ had the extra-pulmonary forms and $18.6 \%(27 / 146) \mathrm{had}$ both forms simultaneously. In Group II, 45.7\% (59/ 129) had histopathological examinations compatible with TB, 38\% (49/129) had a positive bacilloscopy, $7 \%(9 / 129)$ had a positive $M$. tuberculosis culture and in $9.3 \%(12 / 129)$ of the cases the diagnosis was reached through more than one test (Table 5).

In Group II, $43.5 \%$ had the pulmonary form of the disease (56/129), 38.7\% had the extra-pulmonary form (50/129) and 17.8\% (23/129) had both forms simultaneously.

\section{Discussion}

In Brazil, about $5 \%$ of TB cases affect children under 15 years old [6]. This percentage may be even higher when we consider the under-notification and under-diagnosis in this age group due to the paucibacillary form of the disease and the difficulty to obtain the sputum in children under 5 years old [4-5]. TB in children and adolescents often does not follow basic requirements for the diagnosis of infectious diseases, which is the finding of the etiological agent, and it frequently leads to the adoption of therapeutic tests [2-4].

The current series of 275 TB cases in children provided data similar to that from the World Health Organization and from other studies of TB in children that show that there is no effect of gender on predominance [8,14-16] (Table 1).

There was a slightly greater frequency of the disease in the 6 to 10 year age group, when compared with 1 to 5 years, and there was a further increase from 11 to 15 (Table 1). This finding is in accordance with the data provided by the Ministry of Health, from the State of Bahia Department of Health (SESAB) and with the research work done by Benites et al., who studied a similar population in Peru [6-12], and it reinforces the idea that revaccination of school-aged children will reduce the increase in the incidence of this disease among adolescents.

The highest incidence was found in the capital (79.6\%) and most cases (61.2\%) were concentrated in 4 of the 12 districts of the city of Salvador, where the population is made up of low-income residents. This data coincides with the study of Benites et. al in Peru and the one performed by Carneiro and Mota in 
Figure 1. Chest X-ray (frontal view): homogenous alveolar infiltrate in the upper right lobe, showing lobar pneumonia. In this infant, a CAT scan of the chest (not illustrated) showed a group of mediastinal nodes that may lead to the aspiration of the caseous matter they contain.

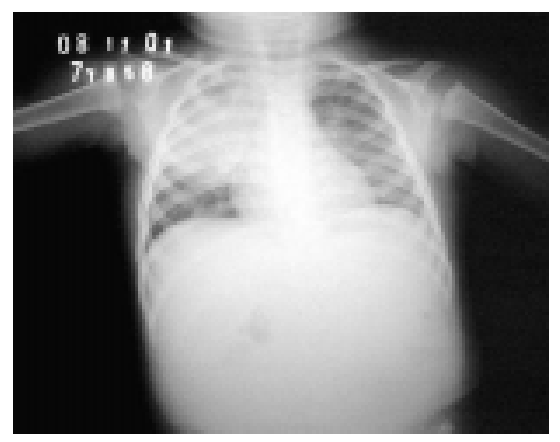

Figure 2. Chest X-ray (frontal view with penetrated mediastinum): large righthilar adenopathy associated with a triangular image of atelectasis of the upper segment of the lower right lobe, secondary to compression of the bronchi by the lymph node.

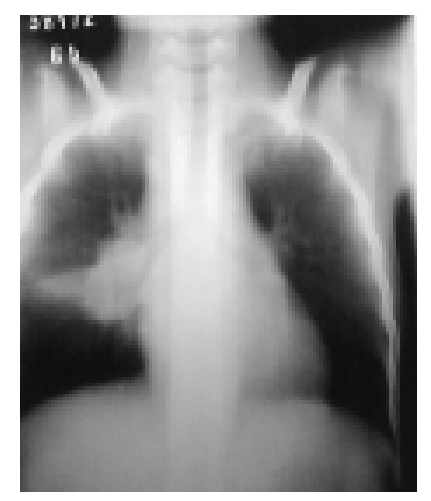

Salvador, Bahia $[12,13]$. In this current series, the predominance of patients from the capital may be due to the greater access to medical services among residents of the metropolitan area of Salvador.

In $63.9 \%$ of the cases, a history of contact with a tuberculosis carrier was detected and this was most frequent in those who were under 5 years old. This data is in accordance with the findings in literature and is due to the fact that young children spend more time confined at home and in close contact with relatives who carry the disease, and therefore are at a higher risk for becoming sick [9].
Figure 3. Chest x-ray (frontal view): upper right mediastinal mass, measuring $5 \mathrm{~cm}$ in diameter. A mediastinoscopic biopsy confirmed the diagnosis of tuberculous adenopathy.

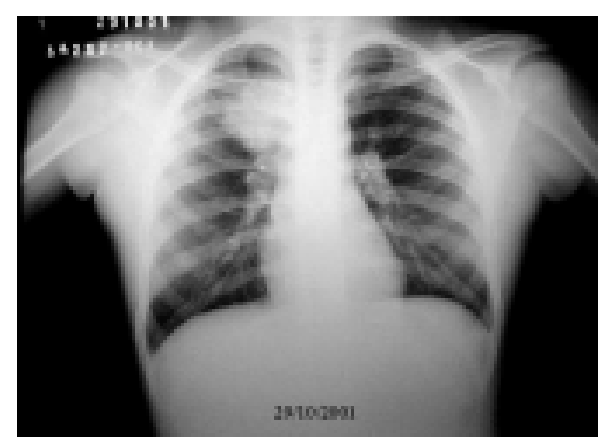

Figure 4. Chest x-ray (frontal view): micronodular infiltrate distributed uniformly in both lungs, showing the miliary pattern of primary tuberculosis.

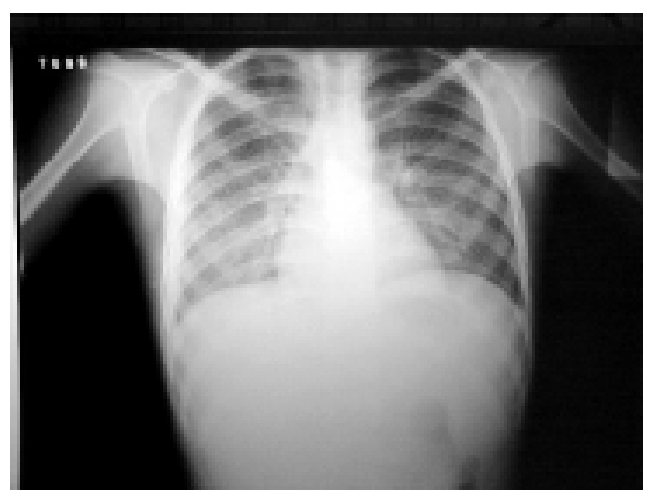

Among those who had medical records, $77.2 \%$ had been vaccinated, which was below the goal determined for 1994 by the Ministry of Health, to vaccinate $90 \%$ of newborns [3-10]. This fact may be because our study started before 1994, a time when vaccination was less frequent in maternity hospitals and also because of children who were not born in maternity hospitals at that time. Among the individuals who were not vaccinated, the majority (79.5\%) resided in Salvador, where health resources are more readily available; this fact makes this data important for an evaluation of vaccination with $\mathrm{BCG}$ in our state. 
Figure 5. Chest x-ray (frontal view): calcified primary complex of tuberculosis in left lung, represented by Ghon focus on the edge of the lower third of the lung and satellite hilar adenopathy. The upper lobe of the right lung shows interstitial infraclavicular infiltration with a retraction of the horizontal fissure, showing the secondary pattern of reactivation of the disease.

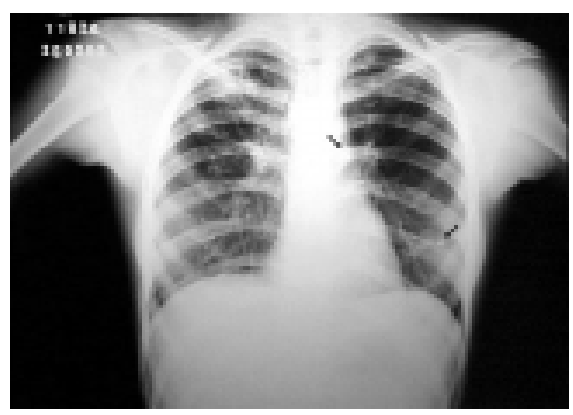

Figure 6. Chest X-ray (frontal view): extensive infiltrate of the right lung with images of heterogeneous alveolar consolidation associated with a thin-walled cavity with an atelectasic component and rectification of the right diaphragm. In the middle third of the left lung, there is a small image of alveolar consolidation. Secondary tuberculosis in a 6-year-old child, showing $M$. tuberculosis infection at a very young age in Bahia.

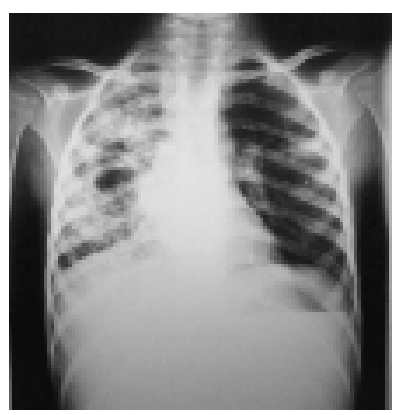

A diagnosis was made in $4.7 \%$ of asymptomatic children thanks to an active search for contacts; this is of great importance to reduce the under-diagnosis index of this disease in Brazil [9]. Sanchez et al. found 38.2\% asymptomatic children in Spain; this finding was attributed to the systematic investigation of contacts carried out in that country [16]. Among symptomatic patients the symptomatology found was the same as observed in adults [3-10].
Figure 7. Two teenagers with multiple suppurating nodes on the right hemithorax, showing chest scrofuloderma, a rare form of skin tuberculosis of the lymph nodes.

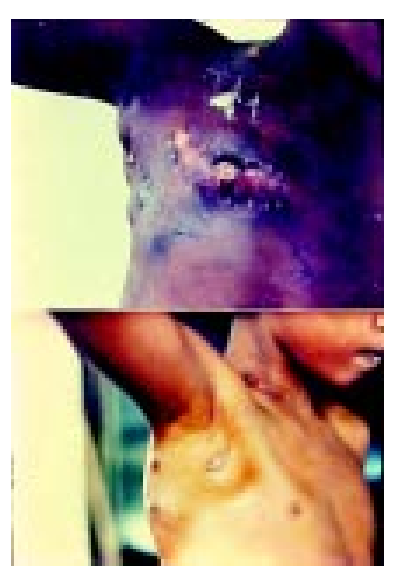

The predominance of the pulmonary form of the disease is described in another series of the disease in children [12,14-16]. There was a high frequency of the extra-pulmonary form of this disease in our series (49.5\%), which differed from the data of the Ministry of Health $(23 \%)$ and the data from SESAB $(25 \%)$. This is due to the fact that in this service there is a systematic investigation of the extra-pulmonary form of the disease and a routine search for the association between this manifestation of TB and the pulmonary forms of the disease. In addition, the formulation of data collection from the Ministry of Health and from SESAB do not contemplate the simultaneous occurrence of pulmonary and extra-pulmonary forms in the same patient, classifying such cases as pulmonary forms and therefore producing incomplete information [6-17].

The simultaneous occurrence in the same patient of pulmonary + extra-pulmonary foci of the disease is common in the primary progressive form of TB. In our study 49 cases $(17.8 \%)$ were found to have this association; this fact was rarely seen in adults, but has been described in other studies of children $[11,16]$.

The interpretation of tuberculin testing in children vaccinated with BCG is of great importance in our population due to the high vaccination coverage. The two relevant aspects in vaccinated children are the 
intensity of the hypersensitivity reaction, which in general is not larger than $15 \mathrm{~mm}$, and the reduction of this reaction two years after vaccination. In individuals infected by M. tuberculosis the intensity of the reaction, besides being greater than in those individuals that are vaccinated, lasts more than 10 years. It has been demonstrated that among children vaccinated with $\mathrm{BCG}$, the risk of becoming sick is higher in those with a PPD reaction $>15 \mathrm{~mm}$ [7].

Tuberculin testing was of great value in this study and it produced a strong reaction in the majority of cases (82.7\%); this percentage was similar to the one found by Sequeira et al. in Argentina, who reported $79 \%$ [11].

Chest X-rays showed a predominance of unilateral right side lesions in children; this is caused by the anatomy of the right main bronchus (more verticalized), which favors the inhalation of the bacillus. Among the most frequent images in this age group, we found the mediastinal/hilar adenopathies, which at times are associated with parenchymal abnormalities, with atelectasis caused by bronchial compression by the lymph node masses, as well as pneumonic infiltrates that cause the caseous content of the lymph node to empty into the bronchus. These images are generally identified in the lower $2 / 3$ of the lung, in contrast with the images of secondary TB in an adult, which are typically in the pulmonary apex. This was a test of special value in the pulmonary forms of the disease, and it was normal in only one case of bronchial TB. It is also useful in extra-pulmonary forms of miliary and pleural TB. In children this test must be performed in all cases of the disease because of the possibility of concomitant lesions in the lungs, even in extrapulmonary forms without respiratory symptoms.

Bacteriological exams contributed very little to diagnosis (different from the case in adults) because some of the children did not undergo them due to the absence of secretion (characteristic of the primary pulmonary form) or because they did not know how to expectorate, especially children under 5 years old. A coincidence was found between the percentage of children who did not undergo sputum bacterioscopy (35.6\%) and the percentage patients between 1 and 5 who did not expectorate (35.3\%); this may explain the low frequency of this exam.

The number of positive cultures was smaller than that of bacilloscopies due to the technical difficulties in the service; not all patients who had a positive bacilloscopy also had their sputum cultured.

Comparing the bacteriological results of our study with other series of similar socioeconomic populations we found that while there were $28 \%$ positive bacilloscopies and/or cultures, Nonino et al. found $8.3 \%$ in the state of Paraná, Brazil, and Sequeira et al. found $10.7 \%$ in Argentina [15-11]. A study carried out by the American CDC indicated $25 \%$ positive cultures and $3 \%$ positive bacilloscopies , for a total of $28 \%$. The total frequency of positive exams was the same as we found; however, in the American study the number of cultures was much higher than that of bacilloscopies, which might have been a consequence of a larger number of patients who had the initial forms of the disease [18].

The histopathological examinations were more useful in the extra-pulmonary forms of the disease; $93.8 \%$ of the biopsied cases had this clinical form of the disease. A positive aspect of our study is that it is the first to describe the clinical and radiological characteristics of TB in children and adolescents in the state of Bahia. Therefore, it can contribute to our knowledge of TB in this age group in this state, help in the process of reaching a diagnosis and reduce the high number of therapeutic tests.

The limitations of this study are a consequence of its retrospective nature, with some loss of information in the medical records; this characteristic rendered the evaluation of the nutritional state impracticable. Another weak point was the absence of HIV serology in children with a non-reactive tuberculin test who had extrapulmonary manifestations of the disease, according to what is recommended currently by the Ministry of Health.

It is also difficult to generalize the results found in this study to the general population in the State of Bahia, since this study was carried out in a reference center that preferentially treats patients who are residents of Salvador. 
In conclusion, there was no gender effect on the predominance of TB, most cases came from lowincome classes who lived in the peripheral areas of the capital, there was a high frequency of cases with contact history with a TB carrier and low vaccination coverage. The symptoms found did not differ from those of adult TB and the most frequent clinical form was primary progressive tuberculosis. Despite all efforts, $51.3 \%$ of patients did not have a bacteriological or histopathological confirmation of their diagnoses.

A strongly reactor tuberculin test result in $82.7 \%$ of the cases and an abnormal chest x-ray in $88.1 \%$ still are the most useful exams for the diagnosis of tuberculosis in children and adolescents in unsophisticated services of low complexity, and they are fairly effective [3,9-15]. In countries with a high prevalence of TB and unfavorable socioeconomic conditions, a factor that could improve actions to handle TB in children is the use of standardized forms with clinical, radiological and epidemiological data; such forms are already in the process of validation by the Brazilian Ministry of Health.

New studies must be directed towards the execution of more cost-effective and more practical diagnostic techniques applicable to children. If rapid diagnostic tests such as PCR were routinely used on the paucibacillary forms of this disease, there would be an enormous improvement in the notification of TB cases in children and adolescents [18].

\section{References}

1. Dye C., Scheele S., Dolin P., et al. Global burden of tuberculosis. Estimated incidence, prevalence and mortality by country. The Journal of the American Medical Association 1999; 282:677-86.

2. Crofton J., Horner N., Miller F. Tuberculosis in children. In Crofton J. Clinical tuberculosis. London: Macmillan, 1992;29-82.

3. Manual de Normas para o Controle da Tuberculose. $4^{\mathrm{a}}$ ed. Brasília. Coordenação de Pneumologia Sanitária, Ministério da Saúde-Brasil, 1995.

4. Sant'Anna C.C., Bethlem N., Alves R. Conduta Diagnóstica em Tuberculose na Criança. J Pneumol 1993;19(2):91-5.
5. Carreira M.N., Sant'Anna C.C. Estudo comparativo de critérios para o diagnóstico de tuberculose em criançs atendidas em centro de saúde. J Pneumol 2000;26:219-26.

6. IBGE. Programa Nacional de Controle da Tuberculose. Instituto Brasileiro de Geografia e Estatística, Brasília, 2002.

7. Sant'Anna C.C. Tuberculose. In: Rozov T. Doenças Pulmonares em Pediatria : Diagnóstico e tratamento. $1^{\mathrm{a}}$ ed. São Paulo: Atheneu, 1999;277-87.

8. WHO. Revised international definitions in tuberculosis control. World Health Organization. Disponível em: http://www.who.int/gtb/publications/index.html.

9. Lemos A.C.M.L. Prevalência e risco de tuberculose em contatos domiciliares. Tese de Doutorado. Universidade Federal da Bahia, Salvador, 183p.,2001

10. I Consenso Brasileiro de Tuberculose. Publicação Oficial da Sociedade Brasileira de Pneumologia e Tisiologia. Jornal de Pneumologia 1997;23:336-8.

11. Sequeira M., Imaz M., Barrera L., et al. Diagnóstico de la tuberculose infantil en provincias de la Argentina. Medicina (B.Aires) 2000;60(2):170-8.

12. Benites R., Vasquez J., Avila L., Durand J. Aspectos clínicos y epidemiológicos de la tuberculosis infantil en el hospital base chimbote - Peru. Diagnóstico 1987;19(6):167-77.

13. Carneiro N., Mota E., Tuberculose em Salvador-Bahia: incidência e algumas variáveis sócio-demográficas em 1980 Rev. Baiana Saúde Publ.,13(1/3):68-80, jan 1986.

14. Castro L., Xavier S. Tuberculose em um hospital infantil de Fortaleza. In: Anais do IX Congresso Brasileiro de Pneumologia Pediátrica. Florianópolis 134p., 2001.

15. Nonino J., Minetto J., Nonino L. Tuberculose na InfânciaEstudo de 36 casos In: Anais do IX Congresso Brasileiro de Pneumologia Pediátrica. Florianópolis 130p, 2001.

16. Sánchez I., Baquero F., Castillo F. Twenty years of pulmonary tuberculosis in children: what has changed? Infectious Diseases Unit, Children's Hospital La Paz, Madrid-Spain Aug. 2001.

17. SESAB, Secretaria de Saúde do Estado da Bahia, Notificação da Tuberculose no Estado, 2002.

18. Natal S.R., Gerardt G.Tuberculose nos menores de 15 anos. Pulmão 1991;1(3):103-7.

19.Chaulk P., Khoo L., Matuszak D. Case Characteristics and Trends in Pediatric Tuberculosis. Public Health Reports 1997;2(112):146-52. 